\title{
Feasibility of Multiparametric Imaging with PET/MR in Head and Neck Squamous Cell Carcinoma
}

\author{
Jacob H. Rasmussen ${ }^{1,2}$, Martin Nørgaard ${ }^{3,4}$, Adam E. Hansen ${ }^{3}$, Ivan R. Vogelius ${ }^{1}$, Marianne C. Aznar ${ }^{1}$, \\ Helle H. Johannesen ${ }^{3}$, Junia Costa ${ }^{3}$, Astrid M.E. Engberg ${ }^{3}$, Andreas Kjær ${ }^{3,5}$, Lena Specht ${ }^{1}$, and Barbara M. Fischer ${ }^{3}$ \\ ${ }^{I}$ Department of Oncology, Section Radiotherapy, Rigshospitalet, University of Copenhagen, Copenhagen, Denmark; ${ }^{2}$ Department \\ of Otorhinolaryngology, Head \& Neck Surgery and Audiology, Rigshospitalet, University of Copenhagen, Copenhagen, Denmark; \\ ${ }^{3}$ Department of Clinical Physiology, Nuclear Medicine and PET, Rigshospitalet, University of Copenhagen, Copenhagen, Denmark; \\ ${ }^{4}$ Neurobiology Research Unit, Rigshospitalet, University of Copenhagen, Copenhagen, Denmark; and ${ }^{5}$ Cluster for Molecular \\ Imaging, University of Copenhagen, Copenhagen, Denmark
}

\begin{abstract}
The purpose of this study was to investigate and assess the correlation and reproducibility of multiparametric imaging in head and neck cancer patients. Methods: Twenty-one patients were included in this prospective scan-rescan study. All patients were scanned twice on an integrated PET and MRI scanner. Gross tumor volumes were defined on T2-weighted MR images, and volumes of interest were defined on diffusion-weighted MRI and ${ }^{18} \mathrm{~F}-\mathrm{FDG} \mathrm{PET}$ $\left(\mathrm{VOI}_{\mathrm{DWI}}, \mathrm{VOI}_{\mathrm{PET}}\right)$. Overlap between volumes was assessed as a percentwise overlap. ${ }^{18} \mathrm{~F}-\mathrm{FDG}$ uptake and diffusion were measured using SUV and apparent diffusion coefficient, and correlation was tested across and within patients and as a voxel-by-voxel analysis. Results: Seventeen patients were available for correlation analysis, and 12 patients were available for assessment of tumor overlap. The median tumor overlap between $\mathrm{VOI}_{\mathrm{DWI}}$ and $\mathrm{VOI} \mathrm{I}_{\mathrm{PET}}$ was $82 \%$ $\left(\mathrm{VOI}_{\mathrm{DWI}}\right.$ in $\left.\mathrm{VOI}_{\mathrm{PET}}\right)$ and $62 \%\left(\mathrm{VOI}_{\mathrm{PET}}\right.$ in $\left.\mathrm{VOI}_{\mathrm{DWI}}\right)$ on scan 1 and scan 2 , respectively. Across patients, the correlation between SUV and apparent diffusion coefficient was weak and nonsignificant. However, in individual patients a weak but significant correlation was identified on a voxel-by-voxel basis. Conclusion: In multiparametric imaging with the integrated PET/MR scanner, the VOIs from DWI and ${ }^{18} \mathrm{~F}$-FDG PET were both within the target volume for radiotherapy and overlapped substantially although not completely. No correlation between ${ }^{18} \mathrm{~F}-\mathrm{FDG}$ uptake and DWI could be found across patients, but within individual patients a statistically significant, but weak, voxel-by-voxel correlation was found. The findings suggest that information on glucose uptake and diffusion coefficient carries complementary information of interest that may be relevant for radiotherapy treatment planning.
\end{abstract}

Key Words: oncology; head and neck; PET/MRI; other; head and neck cancer; multiparametric imaging

J Nucl Med 2017; 58:69-74

DOI: 10.2967/jnumed.116.180091

Received Jul. 4, 2016; revision accepted Aug. 18, 2016.

For correspondence or reprints contact: Jacob H. Rasmussen, Department of Otorhinolaryngology, Head \& Neck Surgery and Audiology, Rigshospitalet F-2074, Blegdamsvej 9, 2100 Copenhagen, Denmark.

E-mail: jacobrasmu@gmail.com

Published online Sep. 8, 2016.

COPYRIGHT @ 2017 by the Society of Nuclear Medicine and Molecular Imaging.
$\mathbf{T}$ he concept of personalized medicine has brought increased awareness to inter- and intratumor heterogeneity (1), and the evidence supporting the importance of considering tumor heterogeneity in therapy planning is increasing (2-4). Single tumor biopsies cannot detect all intratumor heterogeneity, and it is not realistic to perform multiple biopsies of individual tumors or to biopsy all lesions in individual patients with metastatic disease in an attempt to assess intra- or intertumor heterogeneity. Therefore, a new diagnostic method, which can provide a more comprehensive view of the tumor biology, including intratumor heterogeneity, is warranted. Tumor heterogeneity can now be investigated with multiparametric imaging $(5,6)$, and in this respect hybrid-imaging with PET/MR (integrated PET and MRI) provides a promising and noninvasive alternative (7-9).

Squamous cell carcinoma of the head and neck (HNSCC) is a heterogeneous disease (10-12), and with the introduction of PET/ MR imaging it is now possible to assess some of this heterogeneity by comparing 2 functional imaging techniques simultaneously on a voxel-by-voxel basis. For example, comparisons can be made between diffusion-weighted imaging (DWI) and ${ }^{18} \mathrm{~F}-\mathrm{FDG}$, both of which can be quantified, DWI as apparent diffusion coefficient (ADC) and ${ }^{18} \mathrm{~F}$-FDG uptake as the SUV.

Low ADC and high SUV have both been reported to correlate with poor patient outcome $(13,14)$. DWI and ${ }^{18}$ F-FDG PET can potentially offer complementary information in $\operatorname{HNSCC}(15,16)$, and it is hypothesized that biologically different subvolumes can be identified with functional imaging and targeted with radiation dose painting (17). Integrated PET/MR can provide DWI and ${ }^{18} \mathrm{~F}$ FDG uptake, simultaneously reducing misalignment due to time, positioning, and biologic changes, thus allowing assessment of several relevant dose-painting targets and the concordance between such targets. Several studies have stated that ADC and SUV may be important parameters in describing heterogeneity and defining subvolumes within gross tumor volume (GTV) for dose painting. Both PET and DWI may reflect various functional properties of tumor tissue, although this approach obviously requires that the subvolumes are stable over time and lie within the actual target volume. The correlation between ADC and SUV has been explored by several research groups and for various tumor types. In general, there is a consensus that a negative correlation between the minimum ADC value and the $\mathrm{SUV}_{\max }$ exists, which can be used to distinguish between benign and malignant tumors (18-20). Schmidt et al. (21) suggested that a voxelwise correlation 
of ADC and ${ }^{18} \mathrm{~F}$-FDG PET using PET/MR might provide a more sophisticated spatial characterization of tumors, as did Metz et al. in 2015 (22). To the best of our knowledge, this is the first study evaluating the correlation between DWI and ${ }^{18}$ F-FDG PET on a voxelwise basis as well as scan-rescan stability on an integrated PET/MR scanner in patients with HNSCC.

The hypothesis of this study is that there is a strong and reproducible correlation between ${ }^{18} \mathrm{~F}$-FDG PET and DWI both by ADC/SUV measures and by volume of interest (VOI). Here, strong should be interpreted as a sufficiently reliable correlation to allow assessment of ADC from the SUV in a voxel and vice versa with sufficient accuracy for clinical use. The following 4 specific aims are addressed to test the hypothesis of the study: investigate and quantify the overlap between the tumor volumes defined by DWI and ${ }^{18} \mathrm{~F}$-FDG PET images simultaneously on an integrated PET/MR scanner and report the reproducibility, investigate the correlation between ${ }^{18} \mathrm{~F}-\mathrm{FDG}$ uptake (SUV) and $\mathrm{ADC}$ across patients, investigate the voxelwise correlation of ${ }^{18} \mathrm{~F}-\mathrm{FDG}$ uptake and $\mathrm{ADC}$, and explore the potential of an unsupervised cluster analysis of joint ${ }^{18} \mathrm{~F}-\mathrm{FDG}$ and $\mathrm{ADC}$ data to identify tumor subvolumes.

\section{MATERIALS AND METHODS}

\section{Patients}

Twenty-one patients were included in this prospective scan-rescan study after written informed consent was obtained. The patients were scanned twice with exactly $3 \mathrm{~d}$ between scans. All patients in the present study were from a patient cohort of 30 patients originally included in a study designed to test reproducibility of ${ }^{18} \mathrm{~F}-\mathrm{FDG}$ uptake on both PET/CT and PET/MR (23). The study was approved by the local ethics committee (approval no. H-3-2012-072).

\section{${ }^{18}$ F-FDG PET/MR}

All patients were scanned on the same integrated PET/MR system (Biograph mMR; Siemens) (24) with a 3-T magnet using a head and neck coil. Patients were instructed to fast for a minimum of $6 \mathrm{~h}$ and scanned $100-120 \mathrm{~min}$ after injection of ${ }^{18} \mathrm{~F}-\mathrm{FDG}(4 \mathrm{MBq} / \mathrm{kg})$. For the time interval between ${ }^{18} \mathrm{~F}-\mathrm{FDG}$ injection and time to scan on the second scanning day, we attempted to match the actual time interval on the first scanning day.

PET was performed as a single-bed, 20-min acquisition. PET data were reconstructed using ordinary Poisson ordered-subset expectation maximization with 3 iterations, 24 subsets, and $4 \mathrm{~mm}$ gaussian postprocessing filter into $344 \times 344$ matrices. Resolution modeling (point spread function) was not applied.

A T2-weighted sequence was acquired, followed by a Dixon volumetric interpolated breath-hold examination sequence for attenuation correction. All scans were obtained without a gadolinium contrast agent. The imaging protocol for the DWI consisted of a 2-dimensional echoplanar sequence (echo planar imaging) using a matrix size of $92 \times$ $92 \times 24$, voxel size of $2.71 \times 2.71 \times 4 \mathrm{~mm}$, repetition time/echo time of $3,000 / 84 \mathrm{~ms}$, parallel imaging factor (generalized autocalibrating partial parallel acquisition) of 4 , and flip angle of $90^{\circ}$, and was performed in all patients with 3 different b-values $\left(0,500,1,000 \mathrm{~s} / \mathrm{mm}^{2}\right)$. DWI was measured as 3 stacks, each at isocenter to maximize field homogeneity. DWI had an effective echo spacing of $0.145 \mathrm{~ms}$, and DWI distortions were corrected using the algorithm (FMRIB's Utility for Geometrically Unwarping EPIs [where FMRIB is Oxford Centre for Functional MRI of the Brain] [FUGUE]) of the FSL software package (Analysis Group, FMRIB) (25).

\section{Definitions of VOI}

A region of interest covering the primary $\mathrm{T}$ site was defined on MR and PET, respectively. The PET images were assessed by a specialist in nuclear medicine, and the ${ }^{18} \mathrm{~F}$-FDG PET-positive tumor volume, that is, $\mathrm{VOI}_{\mathrm{PET}}$, was defined and delineated manually. This was done by a visual adaptation of an SUV isocontour starting at $40 \%$ of the $\mathrm{SUV}_{\max }$ so as to include the steepest gradient of SUV-avid tissue and exclude physiologic ${ }^{18}$ F-FDG uptake (26).

The DWI was assessed by an MR radiologist. Before the delineation of the VOI defined from DWI ( $\left.\mathrm{VOI}_{\mathrm{DWI}}\right)$, the PET and anatomic images were assessed to exclude patients with a large signal drop out or other severe artifacts on DWI. An example of signal drop out can be seen in Supplemental Figure 1 (supplemental materials are available at http://jnm.snmjournals.org). The radiologist did not have access to the PET imaging during the actual delineation of the $\mathrm{VOI}_{\mathrm{DwI}}$; likewise, the specialist in nuclear medicine did not have access to the MR scan during the delineation.

The definition and delineation of the anatomic GTV on MR $\left(\mathrm{GTV}_{\mathrm{T} 2}\right)$ were performed by a radiologist specializing in tumor delineation. The delineation was performed on the T2-weighted images without access to or prior evaluation of the functional images (i.e., both PET and DWI). The tumor volume was delineated as a target volume for radiotherapy, that is, if there was doubt regarding tumor extension it was included in the $\mathrm{GTV}_{\mathrm{T} 2}$. The MR-defined target volume $\left(\mathrm{GTV}_{\mathrm{T} 2}\right)$ was chosen to ensure that the volumes defined from ${ }^{18} \mathrm{~F}$-FDG PET and DWI were within the relevant target volume for radiotherapy.

\section{Analysis of DWI and PET Data}

SUV, ADC, and volume metrics were extracted from the software package Mirada XD3 (MIRADA Medical). Tumor volume overlap between the respective imaging modalities was assessed as a percentwise overlap.

In addition, a voxelwise analysis of PET, T2-weighted MR images (T2-MRI), and DWI was executed using MATLAB R2014b (8.4.0.150421; 64 bit [The MathWorks]). PET and T2-MRI were initially resampled to the same dimensions as the ADC sequence $(92 \times$ $92 \times 24$ voxels; voxel spacing, $2.71 \times 2.71 \times 4 \mathrm{~mm}$ ) in OsiriX (version 5.7.1; 32 bit), so the dimensions of the PET, T2-MRI, and ADC sequences were all in accordance before a voxelwise analysis. PET and DWI VOIs were imported from Mirada and resampled to the ADC sequence. All voxels present in the delineated VOIs were used in the voxelwise analysis. A gaussian mixture model was used as a datadriven cluster analysis to cluster-specific voxels according to their likelihood of belonging to a certain subvolume (e.g., a multivariate normal distribution), as previously described by Schmidt et al. (21). In our specific context, it was assumed that a small area in the tumor (voxel) belonged to a certain subvolume (e.g., representing underlying biologic tissue characteristics, such as inflammation, necrosis, malignant tissue, benign tissue) defined by a multivariate normal distribution with cluster-specific mean and covariance. On visual assessment, the tumors did not appear heterogeneous and thus the analysis was restricted to search for 2 clusters, and the cluster with the highest SUV was called cluster 2 .

\section{Statistical Analysis}

Statistical analysis was performed in SPSS (version 19; IBM). The reproducibility of SUV and ADC values was assessed using the paired $t$ test. Correlations between $\mathrm{SUV}_{\text {max }}$ and $\mathrm{ADC}_{\text {min }}$ and $\mathrm{SUV}_{\text {mean }}$ and $\mathrm{ADC}_{\text {mean }}$, respectively, on both scans were assessed using Pearson correlation. Correlation of the distribution of ADC and SUV in a voxelwise comparison was assessed using Spearman rank correlation.

\section{RESULTS}

\section{Patients}

Twenty-one patients were scanned twice with PET/MR, as previously described (23). Four patients had to be excluded because of a large signal drop out at the tumor site on the DWI caused by geometric distortion (Supplemental Fig. 1), leaving 17 patients available for further analysis of correlation between SUV and ADC. After the FUGUE correction, no patients had to be excluded because of 


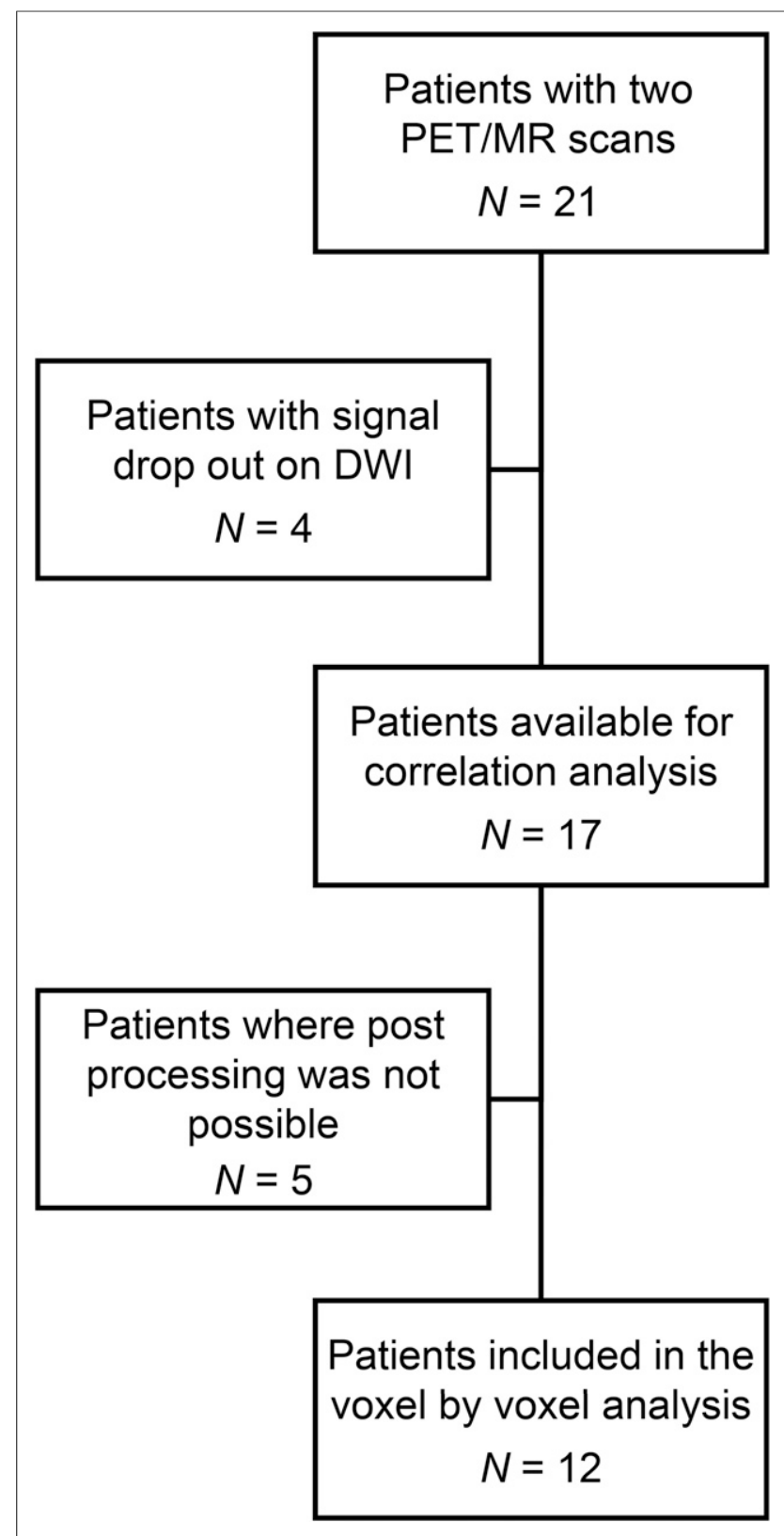

FIGURE 1. Flowchart of patients included.

signal drop out. In 4 other patients, FUGUE correction was not possible in either of the 2 scans and in another 2 patients FUGUE correction was possible only in 1 of the 2 scans, leaving 12 patients available for assessment of tumor overlap between ${ }^{18} \mathrm{~F}$-FDG PET and DWI and for the voxel-by-voxel comparison $(11+1$ with FUGUE correction from the first scan and $11+$ the other with FUGUE correction data from the second scan). The reproducibility of tumor overlaps and voxelwise comparison was therefore feasible in 11 patients who had all data available from both PET/MR scans. A flowchart of patient inclusion can be seen in Figure 1, and Table 1 summarizes the number of patients with data available from the first and second scans.

\section{Overlap of Tumor Volumes}

The volumes from the 3 imaging modalities measured on scans 1 and 2, respectively, are illustrated in Figure 2. The median tumor
TABLE 1

Patients with Data Available from First and Second Scan in Regards to PET, ADC, and Postprocessing (FUGUE), Which Is Necessary to Perform Voxel-by-Voxel Comparison

\begin{tabular}{lcc}
\hline Data & $\begin{array}{c}\text { No. of patients } \\
\text { from first } \\
\text { scan }\end{array}$ & $\begin{array}{c}\text { No. of patients } \\
\text { from second } \\
\text { scan }\end{array}$ \\
\hline For PET & 17 & 17 \\
\hline For ADC & 17 & 17 \\
\hline $\begin{array}{c}\text { For voxel-by-voxel } \\
\text { analysis }\end{array}$ & 12 & 12 \\
\hline
\end{tabular}

volume and the median tumor overlap between the 3 different imaging modalities can be seen in Table 2. For all cases $\mathrm{VOI}_{\mathrm{DWI}}<$ $\mathrm{VOI}_{\mathrm{PET}}<\mathrm{GTV}_{\mathrm{T} 2}$. As expected, the GTV assessed with both DWI $\left(\mathrm{VOI}_{\mathrm{DWI}}\right)$ and ${ }^{18} \mathrm{~F}-\mathrm{FDG}$ PET $\left(\mathrm{VOI}_{\mathrm{PET}}\right)$ was encompassed in the anatomically defined tumor volume $\left(\mathrm{GTV}_{\mathrm{T} 2}\right)$ on both the first and the second scan, respectively (98\% and 99\%) (Table 2). In all but 1 case, more than $73 \%$ of the tumor volume defined from DWI was encompassed in $\mathrm{VOI}_{\mathrm{PET}}$. In the remaining case, only $58 \%$ and $48 \%$ of $\mathrm{VOI}_{\mathrm{DWI}}$ was encompassed in $\mathrm{VOI}_{\mathrm{PET}}$ on the first and second scans, respectively. The median tumor overlap was above $80 \%\left(\mathrm{VOI}_{\mathrm{DWI}}\right.$ within $\left.\mathrm{VOI}_{\mathrm{PET}}\right)$ on both scans 1 and 2 and proved to be reproducible (Table 2). The difference in overlap from scan 1 to scan 2 was small (average 3\%). Two examples of mismatch of $\mathrm{VOI}_{\mathrm{DWI}}$ and $\mathrm{VOI}_{\mathrm{PET}}$ can be seen in Figure 3: a worst case (Fig. 3A) and a representative case (Fig. 3B). Because of the relatively small tumor volume in this patient, even a small mismatch between T2, DWI, and PET has a relatively large percentwise impact in the overlap analysis.

\section{Correlation Between SUV and ADC}

A scatterplot of $\mathrm{SUV}_{\max }$ and $\mathrm{ADC}_{\text {min }}$ can be seen in Figure 4, from both the first scan (Fig. 4A) and the second scan (Fig. 4B). There were no significant differences in $\mathrm{SUV}_{\max }$ (mean difference, $0.9 ; P=0.11$ ) or $\mathrm{ADC}_{\min }$ (mean difference, 9.7; $P=0.69$ ) between

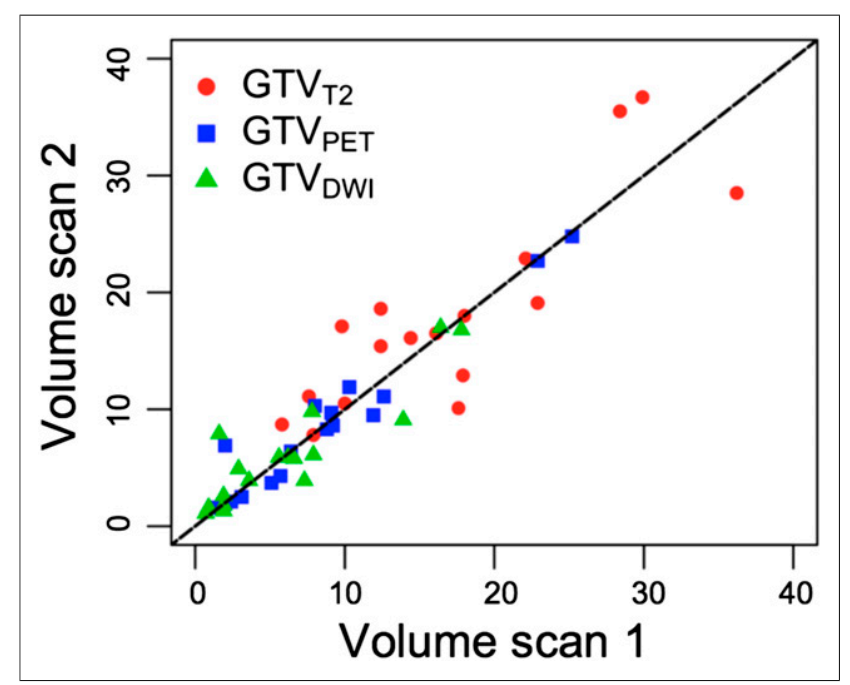

FIGURE 2. Tumor volumes measured with $\mathrm{VOI}_{\mathrm{DW}}$, $\mathrm{VOI}_{\mathrm{PET}}$, and $\mathrm{GTV}_{\mathrm{T} 2}$ on the 2 PET/MR scans. 
TABLE 2

Median Tumor Volume from DWI, ${ }^{18} \mathrm{~F}-\mathrm{FDG}$ PET, and Anatomic T2-Weighted MR

\begin{tabular}{|c|c|c|}
\hline VOI/GTV & Scan 1 median & Scan 2 median \\
\hline $\mathrm{VOI}_{\mathrm{PET}}\left(\mathrm{cm}^{3}\right)$ & $8.0(1.2-25.2)$ & $8.3(1.6-24.8)$ \\
\hline $\mathrm{VOI}_{\mathrm{DWI}}\left(\mathrm{cm}^{3}\right)$ & $5.6(0.7-17.8)$ & $5.8(1.1-17.0)$ \\
\hline $\mathrm{GTV}_{\mathrm{T} 2}\left(\mathrm{~cm}^{3}\right)$ & $16.1(5.8-36.2)$ & $16.5(7.8-36.7)$ \\
\hline $\mathrm{VOI}_{\mathrm{PET}}$ in $\mathrm{VOI}_{\mathrm{DWI}}(\%)$ & $61.5(40.0-86.0)$ & $59.5(42.0-79.0)$ \\
\hline $\mathrm{VOI}_{\mathrm{DWI}}$ in $\mathrm{VOI}_{\mathrm{PET}}(\%)$ & $81.5(58.0-94.0)$ & $84.5(48.0-100)$ \\
\hline $\mathrm{VOI}_{\mathrm{DW}}$ in $\mathrm{GTV}_{\mathrm{T} 2}(\%)$ & $97.0(82.0-100)$ & $98.0(84.0-100)$ \\
\hline $\mathrm{VOI}_{\mathrm{PET}}$ in $\mathrm{GTV}_{\mathrm{T} 2}(\%)$ & $97.5(90.0-100)$ & $99.5(89.0-100)$ \\
\hline
\end{tabular}

Data in parentheses are ranges.

the first and the second scans. On a tumor basis, the correlation between $\mathrm{SUV}_{\max }$ and $\mathrm{ADC}_{\min }$ was not significant, with correlation coefficients of $-0.26(P=0.32)$ and $-0.37(P=0.15)$ on scans 1 and 2 , respectively. Similarly, no significant difference between $\mathrm{SUV}_{\text {mean }}$ and $\mathrm{ADC}_{\text {mean }}$ was observed $(P=0.9$ and 0.5$)$ and the correlation was not significant $(-0.05[P=0.85]$ and $-0.39[P=0.13])$.

The correlation between ADC and SUV on a voxelwise level was significantly negative in 9 of 12 patients on the first scan. However, the correlation coefficients were small, suggesting only a weak correlation (mean correlation coefficient $\rho,-0.23$ [range, -0.63 to 0.31$]$ ) including data from all 22 scans).

\section{Cluster Analysis}

The voxel-by-voxel comparison and the cluster analysis proved feasible and in most of the 11 cases reproducible (assessed visually). All results can be seen in Table 3 and in the supplemental materials (Supplemental Fig. 2).

Cluster 2 (red) encompassed most of the VOI $\mathrm{VET}_{\text {(median over- }}$ lap of $91 \%$ and $97 \%$ on the first and second scans, respectively) and most $\mathrm{VOI}_{\mathrm{DWI}}$ (median overlap of $65 \%$ and $52 \%$ on the first

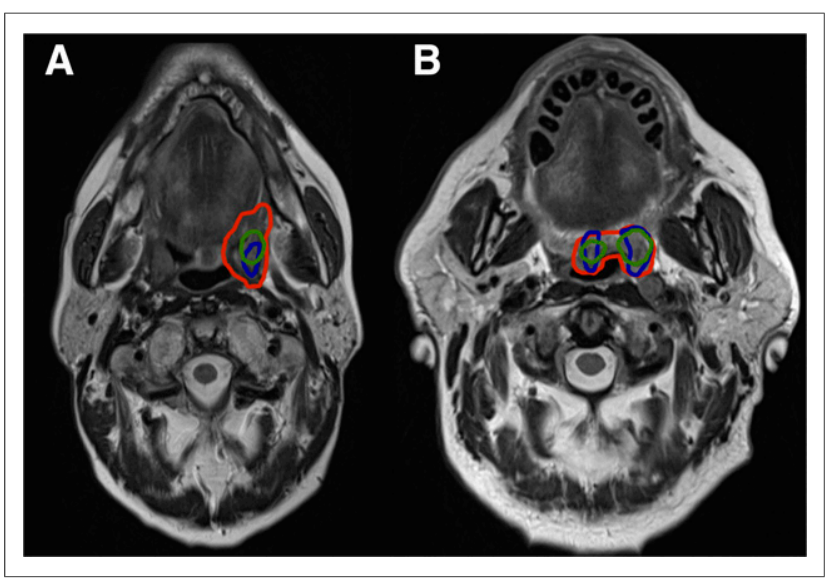

FIGURE 3. Examples of tumor overlap between tumor defined from DWI (blue contour), ${ }^{18} \mathrm{~F}-\mathrm{FDG}$ PET/MR (green contour), and T2-weighted MR (red contour). (A) Worst case of overlap between $\mathrm{VOI}_{\mathrm{DWI}}$ and VOIPET. Forty-two percent of $\mathrm{VOI}_{\mathrm{PET}}$ is within $\mathrm{VOI}_{\mathrm{DWl}}$, and $63 \%$ of $\mathrm{VOI}_{\mathrm{DWI}}$ is within VOI $\mathrm{PET}_{\text {. }}$ (B) Representative case in which $67 \%$ of $\mathrm{VOI}_{\mathrm{PET}}$ is within $\mathrm{VOI}_{\mathrm{DWI}}$, and $68 \%$ of $\mathrm{VOI}_{\mathrm{DWI}}$ is within $\mathrm{VOI}_{\mathrm{PET}}$. and second scan, respectively). Likewise, cluster 1 encompassed only a small part of $\mathrm{VOI}_{\mathrm{DWI}}$ and $\mathrm{VOI}_{\mathrm{PET}}$ on both scans (Table 3). In most but not all patients, separation between clusters appeared to be defined by an SUV threshold and hence not dependent on ADC (Supplemental Fig. 2).

In Figure 5, slices from the PET and the DWI scans from the same 2 patients as in Figure 3 as well as the correlation plots from the voxel-by-voxel analysis can be seen. The representative overlap case (Figs. $5 \mathrm{E}-5 \mathrm{H}$ ) has clusters defined primarily by an SUV around 6. For the poor overlap case (Figs. 5A-5D), conversely, the clusters are not separated by a single SUV but depend on both SUV and ADC in a nontrivial manner.

\section{DISCUSSION}

The tumor overlap between $\mathrm{VOI}_{\mathrm{DWI}}$ and $\mathrm{VOI}_{\mathrm{PET}}$ was not complete but substantial and reproducible, with most $\mathrm{VOI}_{\mathrm{DWI}}$ encompassed in $\mathrm{VOI}_{\mathrm{PET}}(>80 \%)$ and most $\mathrm{VOI}_{\mathrm{PET}}$ encompassed in $\mathrm{VOI}_{\mathrm{DWI}}(>60 \%)$ on both scans. There was no significant correlation between the quantifiable measures $\mathrm{SUV}_{\max }$ and $\mathrm{ADC}_{\min }$ across patients; however, on a voxel-by-voxel basis the correlation between ADC and SUV was significant, albeit probably too weak to allow any meaningful assessment of one image metric from the other. The cluster analysis proved feasible and reproducible, identifying a volume encompassing most $\mathrm{VOI}_{\mathrm{PET}}$ and a smaller part of VOI $_{\text {DWI. }}$.

With the integrated PET/MR scanner, the tumor is assessed simultaneously, and the head and neck coil used limits the movement of the patient during scanning. Hence, it is unlikely that a misalignment between the DWI and PET images is the main reason for discrepancy between the volumes $\mathrm{VOI}_{\mathrm{PET}}$ and $\mathrm{VOI}_{\mathrm{DWI}}$. Other technical reasons for the partial overlap include geometric distortions of the DWI signal close to air cavities such as in the pharynx, which may remain despite the optimized DWI acquisition and FUGUE correction. Both image acquisition and postprocessing were optimized to minimize distortions. The visually judged correspondence of DWI and ${ }^{18} \mathrm{~F}-\mathrm{FDG}$ PET was overall good, as seen in the figures (25). After this FUGUE correction was implemented, no patient had to be excluded because of signal drop out (Supplemental Fig. 1). The remaining part of the observed mismatch is likely due to a biologic difference-that is, intratumor heterogeneity. Thus, when the mismatch due to repositioning and technical distortions is minimized, the method presented here might have the potential to identify and describe areas of the tumor with different biologic characteristics, which is a prerequisite for personalized treatment in the future.

Dose painting is a novel concept in radiotherapy in which the radiation dose is escalated in parts of the tumor thought to be treatment resistant such as hypoxic regions or regions with high pretreatment ${ }^{18} \mathrm{~F}$-FDG uptake $(27,28)$. Tumor areas with high glucose uptake or high cellularity are hypothesized as relevant targets for dose painting $(17,29)$, and if the tumor subvolume defined by DWI and ${ }^{18} \mathrm{~F}$-FDG PET is identical it could in theory be possible to replace ${ }^{18} \mathrm{~F}-\mathrm{FDG}$ with another PET tracer without significant loss of information when imaged simultaneously with DWI on an integrated PET/MR scanner. The replacement of ${ }^{18}$ F-FDG with another PET tracer could provide additional potentially important information of tumor biology. For instance, tumor subregions with high proliferation ( $3^{\prime}$-deoxy- $3^{\prime}-{ }^{18} \mathrm{~F}$-fluorothymidine), hypoxia ( ${ }^{18} \mathrm{~F}$-fluoroazomycin arabinoside), or markers for aggressiveness; for example, urokinase-type plasminogen activator receptor PET 


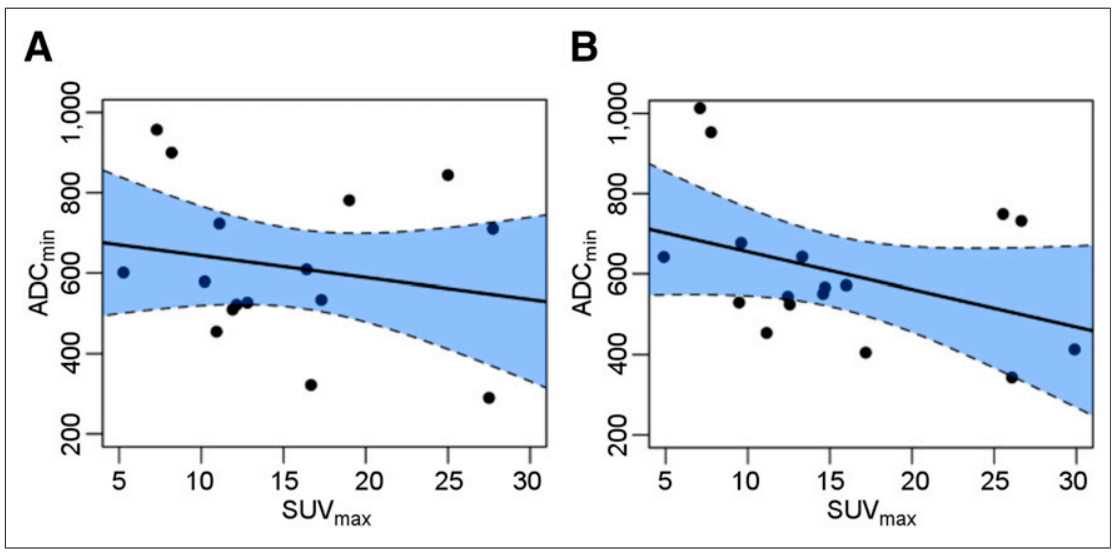

FIGURE 4. Scatterplot of $S U V_{\max }$ and $A D C_{\min }$ from first scan (A) and from second scan (B). Data are fitted to linear model, and laid over with $95 \%$ confidence interval (blue).

TABLE 3

Overlap Between the 2 Clusters and VOI Defined from DWI and ${ }^{18}$ F-FDG PET/MR Is Reported in Percentage

\begin{tabular}{|c|c|c|}
\hline Volume overlap & Scan 1 median & Scan 2 median \\
\hline Cluster 1 in $\mathrm{VOI}_{\mathrm{DWI}}(\%)$ & $14.4(0.1-32.1)$ & $12.6(0.4-24.6)$ \\
\hline Cluster 1 in $\mathrm{VOI}_{\mathrm{PET}}(\%)$ & $14.6(2.6-45.6)$ & $13.8(0-60.2)$ \\
\hline Cluster 2 in $\mathrm{VOI}_{\mathrm{DWI}}(\%)$ & $65.4(33.7-82.1)$ & $51.8(28.8-88.6)$ \\
\hline Cluster 2 in $\mathrm{VOI}_{\mathrm{PET}}(\%)$ & 90.5 (59.4-97.9) & $97.2(55.7-97.9)$ \\
\hline \multicolumn{3}{|c|}{ Data in parentheses are ranges. } \\
\hline
\end{tabular}

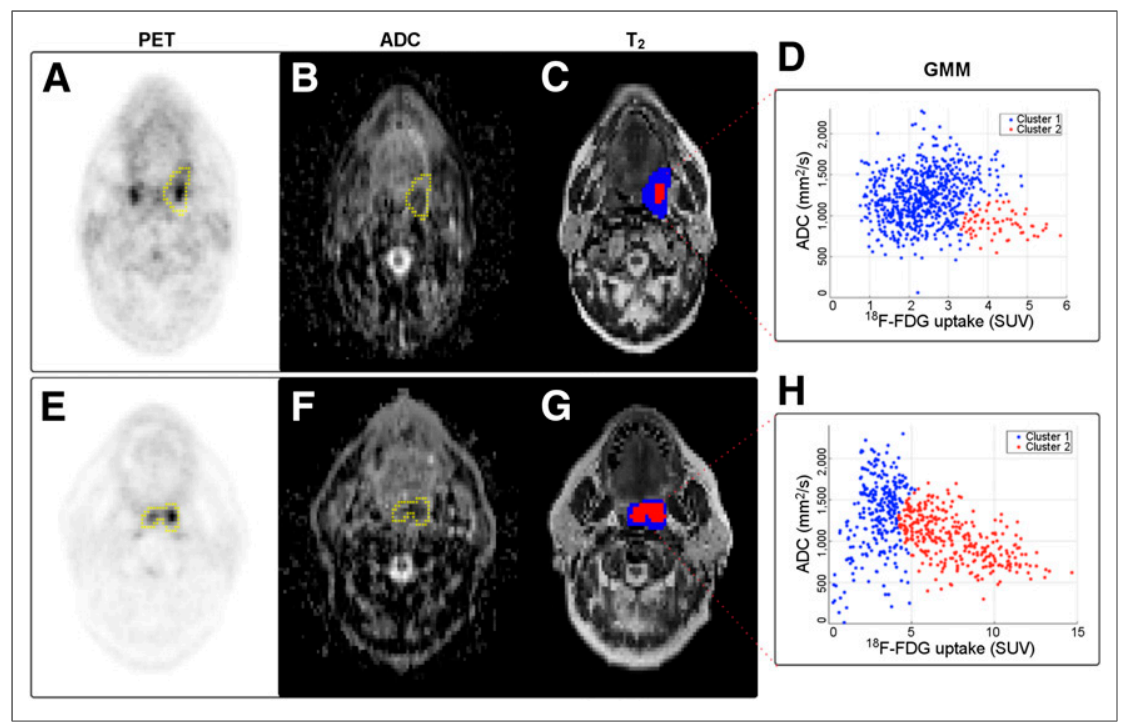

FIGURE 5. PET image (A), image from $A D C$ map (B), and T2-weighted image (C) from patient in Figure 3A (poor overlap case). Yellow contour represents $\mathrm{GTV}_{\mathrm{T} 2}$. (D) Scatterplot from voxel-byvoxel analysis showing respective clustering assignment from cluster analysis. PET image (E), ADC map $(F)$, and T2 image $(G)$ for patient in Figure 3B (representative overlap case). $(H)$ Scatterplot and clustering assignment from cluster analysis.

would be interesting in terms of not only multiparametric imaging research but also potential clinical relevance $(30,31)$. These subregions might be used for treatment guidance such as in dose painting or be used to select patients for other more aggressive treatment regimens in the future. The tumor overlap of $\mathrm{VOI}_{\mathrm{DWI}}$ and $\mathrm{VOI}_{\mathrm{PET}}$ assessed in this study is substantial but nevertheless only partial, and together with the relatively weak correlation between image surrogates of high cellularity (assessed with DWI) and high glucose uptake (assessed with ${ }^{18}$ F-FDG PET), our results indicate that the 2 imaging modalities provide complementary information on tumor biology. Moreover, these subvolumes of the tumor seem to be stable over time with regard to both quantitative ADC and SUV and spatial placement. The reproducibility of the ${ }^{18} \mathrm{~F}-\mathrm{FDG}$ measurements has been documented in another study (23), but the reproducibility of the ADC values has not previously been assessed.

The partial overlap demonstrated in this study is in concordance with the study by Houweling et al. (32). Thus, substituting one modality with the other is not possible without some loss of information. It can also be concluded that replacing ${ }^{18} \mathrm{~F}$-FDG PET with DWI for tumor delineation would yield smaller target volumes in radiotherapy, potentially missing substantial tumor volume as defined by ${ }^{18} \mathrm{~F}-\mathrm{FDG}$ PET. It is also possible that the subvolume identified by the cluster-analysis based on information from both DWI and ${ }^{18} \mathrm{~F}$-FDG PET could be a potential target for dose escalation. But this needs confirmation in studies with histologic correlation or failure pattern analysis (33). It could be argued that the cluster analysis did not seem to provide further useful information compared with the overlap analysis from the manual delineated tumor volumes $\left(\mathrm{VOI}_{\mathrm{PET}}\right.$ and $\left.\mathrm{VOI}_{\text {DWI }}\right)$. Nevertheless, the substantial overlap of information from DWI and ${ }^{18}$ F-FDG PET may be encouraging if non- ${ }^{18}$ F-FDG is to be used as part of PET/MR imaging.

To our knowledge, only 1 study has evaluated the correlation between DWI and ${ }^{18} \mathrm{~F}-\mathrm{FDG}$ PET on an integrated PET/ MR scanner in patients with HNSCC (6). This study also reported a negative correlation, however, the study included only 7 patients with different tumor subsites (6). The existing studies in the literature either are not performed on an integrated PET/MR scanner or do not include patients with head and neck tumors $(21,22,34-38)$. The nonsignificant correlation found across patients suggests that information on glucose metabolism and cellularity from only 1 voxel, the voxel within the tumor either with the highest ${ }^{18} \mathrm{~F}-\mathrm{FDG}$ uptake $\left(\mathrm{SUV}_{\max }\right)$ or with the lowest diffusion $\left(\mathrm{ADC}_{\min }\right)$, may be too crude to adequately describe the tumor and depict a potential correlation. This is substantiated by the significant correlation reported in most cases in the voxel-by-voxel analysis (Supplemental Fig. 2); nevertheless, the weak correlation shows that a biologic mechanistic hypothesis in which diffusion of water is uniformly impaired in areas with high glucose metabolism is an oversimplification. 
Though the results in this study also seem reproducible from scan 1 to scan 2, the small number of patients is a limitation of this study, and there could be true correlations that the sample size is too small to detect. Moreover, the tumors in this patient cohort are relatively small, and the application of voxel-by-voxel analysis for description of tumor heterogeneity will probably be more relevant and provide more information in larger tumors.

\section{CONCLUSION}

In multiparametric imaging with integrated PET/MR, the VOIs from DWI and ${ }^{18}$ F-FDG PET were both within the target volume for radiotherapy and overlapped substantially although not completely. This is important for further research in tumor biology and may have implications for the use of PET/MR for radiotherapy in head and neck cancer and possibly in other malignancies. No significant correlation between ${ }^{18} \mathrm{~F}-\mathrm{FDG}$ uptake and DWI could be found across patients. However, in individual patients a weak correlation could be found on a voxel-by-voxel basis. This suggests that, despite the partial tumor overlap, ${ }^{18}$ F-FDG PET and DWI detect different aspects of tumor biology, and they may possibly have complementary roles in target definition for dose painting, which should be explored further with histologic validation.

\section{DISCLOSURE}

Jacob H. Rasmussen received support from the Arvid Nilssons Foundation. No other potential conflict of interest relevant to this article was reported.

\section{REFERENCES}

1. Vogelstein B, Papadopoulos N, Velculescu VE, Zhou S, Diaz LA, Kinzler KW. Cancer genome landscapes. Science. 2013;339:1546-1558.

2. Gerlinger M, Rowan AJ, Horswell S, et al. Intratumor heterogeneity and branched evolution revealed by multiregion sequencing. N Engl J Med. 2012;366:883-892.

3. Yap TA, Gerlinger M, Futreal PA, Pusztai L, Swanton C. Intratumor heterogeneity: seeing the wood for the trees. Sci Transl Med. 2012;4:127ps10.

4. O'Connor JPB, Rose CJ, Waterton JC, Carano RAD, Parker GJM, Jackson A. Imaging intratumor heterogeneity: role in therapy response, resistance, and clinical outcome. Clin Cancer Res. 2015;21:249-257.

5. Even AJG, De Ruysscher D, Van Elmpt W. The promise of multiparametric imaging in oncology: how do we move forward? Eur J Nucl Med Mol Imaging. 2016;43:1195-1198.

6. Leibfarth S, Simoncic U, Mönnich D, et al. Analysis of pairwise correlations in multi-parametric PET/MR data for biological tumor characterization and treatment individualization strategies. Eur J Nucl Med Mol Imaging. 2016;43:1199-1208.

7. Balyasnikova S, Löfgren J, de Nijs R, Zamogilnaya Y, Højgaard L, Fischer BM. PET/MR in oncology: an introduction with focus on MR and future perspectives for hybrid imaging. Am J Nucl Med Mol Imaging. 2012;2:458-474.

8. Buchbender C, Heusner TA, Lauenstein TC, Bockisch A, Antoch G. Oncologic PET/MRI, part 1: tumors of the brain, head and neck, chest, abdomen, and pelvis. J Nucl Med. 2012;53:928-938.

9. Platzek I, Beuthien-Baumann B, Schneider M, et al. PET/MRI in head and neck cancer: initial experience. Eur J Nucl Med Mol Imaging. 2013;40:6-11.

10. Stransky N, Egloff AM, Tward AD, et al. The mutational landscape of head and neck squamous cell carcinoma. Science. 2011;333:1157-1160.

11. Leemans CR, Braakhuis BJM, Brakenhoff RH. The molecular biology of head and neck cancer. Nat Rev Cancer. 2011;11:9-22.

12. Cancer Genome Atlas Network. Comprehensive genomic characterization of head and neck squamous cell carcinomas. Nature. 2015;517:576-582.

13. Rasmussen JH, Vogelius IR, Fischer BM, et al. Prognostic value of ${ }^{18} \mathrm{~F}$-fludeoxyglucose uptake in 287 patients with head and neck squamous cell carcinoma. Head Neck. 2015;37:1274-1281.

14. Lambrecht $M$, Van Calster B, Vandecaveye V, et al. Integrating pretreatment diffusion weighted MRI into a multivariable prognostic model for head and neck squamous cell carcinoma. Radiother Oncol. 2014;110:429-434.
15. Becker M, Zaidi H. Imaging in head and neck squamous cell carcinoma: the potential role of PET/MRI. Br J Radiol. February 3, 2014 [Epub ahead of print].

16. Kwee TC, Takahara T, Ochiai R, et al. Complementary roles of whole-body diffusion-weighted MRI and ${ }^{18}$ F-FDG PET: the state of the art and potential applications. J Nucl Med. 2010;51:1549-1558.

17. Bentzen SM, Gregoire V. Molecular imaging-based dose painting: a novel paradigm for radiation therapy prescription. Semin Radiat Oncol. 2011;21:101-110.

18. Grueneisen J, Beiderwellen K, Heusch P, et al. Correlation of standardized uptake value and apparent diffusion coefficient in integrated whole-body PET/ MRI of primary and recurrent cervical cancer. PLoS One. 2014;9:e96751.

19. Heusch P, Buchbender C, Köhler J, et al. Correlation of the apparent diffusion coefficient (ADC) with the standardized uptake value (SUV) in hybrid ${ }^{18}$ F-FDG PET/MRI in nonsmall cell lung cancer (NSCLC) lesions: initial results. RöFo. 2013;185:1056-1062.

20. Ho K-C, Lin G, Wang J-J, Lai C-H, Chang C-J, Yen T-C. Correlation of apparent diffusion coefficients measured by 3T diffusion-weighted MRI and SUV from FDG PET/CT in primary cervical cancer. Eur J Nucl Med Mol Imaging. 2009;36:200-208.

21. Schmidt H, Brendle C, Schraml C, et al. Correlation of simultaneously acquired diffusion-weighted imaging and 2-deoxy- $\left[{ }^{18} \mathrm{~F}\right]$ fluoro-2-D-glucose positron emission tomography of pulmonary lesions in a dedicated whole-body magnetic resonance/ positron emission tomography system. Invest Radiol. 2013;48:247-255.

22. Metz S, Ganter C, Lorenzen S, et al. Multiparametric MR and pet imaging of intratumoral biological heterogeneity in patients with metastatic lung cancer using voxel-by-voxel analysis. PLoS One. 2015;10:e132386.

23. Rasmussen JH, Fischer BM, Aznar MC, et al. Reproducibility of FDG PET uptake measurements in head and neck squamous cell carcinoma on both PET/CT and PET/MR. Br J Radiol. 2015;88:20140655.

24. Delso G, Furst S, Jakoby B, et al. Performance measurements of the Siemens mMR integrated whole-body PET/MR scanner. J Nucl Med. 2011;52:1914-1922.

25. Hansen AE, Rasmussen J, Johannesen HH, et al. Geometric distortions of diffusion weighted imaging of the head/neck in combined PET/MR: optimization of image acquisition and post-processing correction for oncology applications [brief communication]. EJNMMI Phys. 2014;1:A76.

26. Bayne M, Hicks RJ, Everitt S, et al. Reproducibility of "intelligent" contouring of gross tumor volume in non-small-cell lung cancer on PET/CT images using a standardized visual method. Int J Radiat Oncol Biol Phys. 2010;77:1151-1157.

27. Bentzen SM. Theragnostic imaging for radiation oncology: dose-painting by numbers. Lancet Oncol. 2005;6:112-117.

28. Tanderup K, Olsen DR, Grau C. Dose painting: art or science? Radiother Oncol. 2006;79:245-248.

29. Dirix P, Vandecaveye V, De Keyzer F, Stroobants S, Hermans R, Nuyts S. Dose painting in radiotherapy for head and neck squamous cell carcinoma: value of repeated functional imaging with ${ }^{18} \mathrm{~F}$-FDG PET, ${ }^{18} \mathrm{~F}$-fluoromisonidazole PET, diffusionweighted MRI, and dynamic contrast-enhanced MRI. J Nucl Med. 2009;50:1020-1027.

30. Bailey DL, Antoch G, Bartenstein P, et al. Combined PET/MR: the real work has just started. Summary report of the Third International Workshop on PET/MR Imaging. Mol Imaging Biol. 2015;17:297-312.

31. Persson M, Skovgaard D, Brandt-Larsen M, et al. First-in-human uPAR PET: imaging of cancer aggressiveness. Theranostics. 2015;5:1303-1316.

32. Houweling AC, Wolf AL, Vogel WV, et al. FDG-PET and diffusion-weighted MRI in head-and-neck cancer patients: implications for dose painting. Radiother Oncol. 2013;106:250-254.

33. Due AK, Vogelius IR, Aznar MC, et al. Recurrences after intensity modulated radiotherapy for head and neck squamous cell carcinoma more likely to originate from regions with high baseline $\left[{ }^{18} \mathrm{~F}\right]-\mathrm{FDG}$ uptake. Radiother Oncol. 2014;111:360-365.

34. Choi SH, Paeng JC, Sohn C-H, et al. Correlation of ${ }^{18} \mathrm{~F}-\mathrm{FDG}$ uptake with apparent diffusion coefficient ratio measured on standard and high $\mathrm{b}$ value diffusion MRI in head and neck cancer. $J$ Nucl Med. 2011;52:1056-1062.

35. Fruehwald-Pallamar J, Czerny C, Mayerhoefer ME, et al. Functional imaging in head and neck squamous cell carcinoma: correlation of PET/CT and diffusionweighted imaging at 3 Tesla. Eur J Nucl Med Mol Imaging. 2011;38:1009-1019.

36. Nakajo M, Kajiya Y, Kaneko T, et al. FDG PET/CT and diffusion-weighted imaging for breast cancer: prognostic value of maximum standardized uptake values and apparent diffusion coefficient values of the primary lesion. Eur J Nucl Med Mol Imaging. 2010;37:2011-2020.

37. Nakamatsu S, Matsusue E, Miyoshi H, Kakite S, Kaminou T, Ogawa T. Correlation of apparent diffusion coefficients measured by diffusion-weighted MR imaging and standardized uptake values from FDG PET/CT in metastatic neck lymph nodes of head and neck squamous cell carcinomas. Clin Imaging. 2012;36:90-97.

38. Varoquaux A, Rager O, Lovblad K-O, et al. Functional imaging of head and neck squamous cell carcinoma with diffusion-weighted MRI and FDG PET/CT: quantitative analysis of ADC and SUV. Eur J Nucl Med Mol Imaging. 2013;40:842-852. 